\begin{tabular}{|c|c|c|c|c|c|c|c|c|c|c|}
\hline 1 & 2 & 3 & 4 & 5 & 6 & 7 & 8 & 9 & IO & I I \\
\hline $\begin{array}{l}\text { Boss } \\
\text { P.G. C. }\end{array}$ & Bez. & Gr. & Spektr. & $\mu_{\text {(saec. })}$ & $p$ & $\lambda$ & $\nu$ ber. & zbeob. & $\pi$ & $\left(\pi={ }^{m} o^{\prime \prime} .1\right)$ \\
\hline 678 & $\operatorname{Pi} 2^{h} 220$ & $5^{m} \cdot 6$ & $\mathrm{~B}_{5}$ & $4: 3$ & $129^{\circ}$ & $96^{\circ}$ & -2.2 & - & 0.009 & $+0^{m} \cdot 4$ \\
\hline 740 & $3 \circ$ Persei & $5 \cdot 5$ & $\mathrm{~B}_{5}$ & 3.8 & I 34 & 89 & +0.4 & - & 0.008 & 0.0 \\
\hline 742 & $29 \gg$ & $5 \cdot 3$ & $\mathrm{~B}_{3}$ & $4 \cdot 5$ & 132 & 93 & - I.I & -0.6 & 0.010 & +0.3 \\
\hline 744 & $3 I »$ & 5.2 & $\mathrm{~B}_{3}$ & 4.2 & I 3 I & 93 & - I.I & - & 0.009 & 0.0 \\
\hline 767 & $\mathrm{Pi} 3^{h} 37$ & 5.4 & $B_{5}$ & 3.6 & I 26 & $9 \mathrm{I}$ & -0.4 & +3.4 & 0.008 & -0.1 \\
\hline 772 & $\alpha$ Persei & 1.7 & $\mathrm{~F}_{5}$ & 3.8 & I 36 & 92 & -0.8 & -2.2 & 0.008 & -3.8 \\
\hline 780 & $\operatorname{Br} 476$ & 5.1 & $\mathrm{~B}_{3}$ & 3.2 & I 29 & 9 I & -0.4 & - & 0.007 & -0.7 \\
\hline 783 & $\mathrm{Pi} 3^{\mathrm{h}} 5^{6}$ & 5.8 & $\mathrm{~B}_{5}$ & 4.9 & 119 & 92 & -0.8 & 一 & $0.01 \mathrm{I}$ & +1.0 \\
\hline 790 & 34 Persei & 4.8 & $\mathrm{~B}_{3}$ & $4 \cdot 4$ & I $3^{8}$ & $9 \mathrm{I}$ & -0.4 & $-\mathrm{r}: 6$ & 0.010 & -0.2 \\
\hline 796 & $\mathrm{Br}_{4} 80$ & 6.1 & B8 & $4 \cdot 7$ & ${ }^{1} 36$ & 90 & 0.0 & - & 0.010 & $+\mathrm{I} . \mathrm{I}$ \\
\hline 8 I 7 & $\psi$ Persei & 4.4 & $\mathrm{~B}_{5}$ & $4 \cdot 3$ & I 27 & 90 & 0.0 & +0.7 & 0.009 & -0.8 \\
\hline 838 & $\delta \gg$ & 3.0 & $\mathrm{~B}_{5}$ & 4.6 & I 36 & 88 & +0.8 & +0.7 & 0.010 & -2.0 \\
\hline 898 & $\mathrm{Pi} 3^{h} 186$ & $5 \cdot 5$ & $\mathrm{~B}_{5}$ & 3.9 & I 28 & 87 & $+\mathrm{I} . \mathrm{I}$ & $(+0.9)$ & 0.008 & 0.0 \\
\hline 9 ז0 & $\varepsilon$ Persei & 2.9 & Bo & $3 \cdot 9$ & I 38 & $8 I$ & $+3 \cdot 4$ & var. & 0.009 & -2.3 \\
\hline 947 & $c \quad 》$ & 4.2 & $\mathrm{~B}_{3}$ & $4 \cdot 4$ & I 33 & 86 & +1.5 & - & 0.010 & -0.8 \\
\hline 1003 & $d$ & $4 \cdot 9$ & $\mathrm{~B}_{3}$ & $4 \cdot 5$ & I 48 & $8_{3}$ & +2.6 & $+3 .-$ & 0.010 & - O.I \\
\hline I 253 & I 5 Camelop. & 6.4 & $\mathrm{~B}_{3}$ & $3 \cdot 5$ & I 4 I & 88 & +0.8 & - & 0.008 & +0.9 \\
\hline 1274 & g Aurigae & $5 \cdot 3$ & $\mathrm{~B}_{3}$ & $4 \cdot 5$ & I 46 & 73 & +6.3 & $(-\mathrm{II})$ & 0.010 & +0.3 \\
\hline
\end{tabular}

Die Ungenauigkeiten der Eigenbewegung und vor allem $\mid$ hellste, $\varepsilon$ Persei, besitzt das Spektrum Bo und der schwächste, die Unsicherheit der Bestimmung der Translationsgeschwindigkeit gehen voll in die Werte von $\pi$ und $m$ ein, weshalb die Tabelle nur einen Überblick geben kann. Ein gutes Kriterium für die Zusammengehörigkeit der Gruppe ist die abnehmende absolute Helligkeit mit fortschreitendem Spektraltypus, mit der eigentümlichen Ausnahme von $\alpha$ Persei. Der absolut Br. 480, schließt mit Spektrum B8. Vermutlich wird noch eine größère Zahl schwächerer Sterne, die nicht im Boss P.G.C. vorhanden sind, der Perseusgruppe angehören. Wegen der Kleinheit der Eigenbewegung dürfte aber ihre Auffindung recht schwierig sein.

Berlin-Babelsberg, 1920 April 8. K.F. Bottlinger.

\title{
Photometrische Kontrolle der auf der Potsdamer und Harvard-Sternwarte beobachteten schwachen Sterne in der Nova Persei-Gegend. Von K. Graff.
}

Bei der Reduktion meiner Beobachtungen der Nova Persei aus den letzten Jahren war mir bei den schwächsten Sternen der starke Unterschied zwischen der Potsdamer $\left.(P)^{1}\right)$ und Harvardskala $(\mathrm{H})^{2}$ ) aufgefallen. Die schwächeren gemeinsam beobachteten Sterne der Hagenschen Folge haben nämlich in den beiden Verzeichnissen die folgenden Helligkeitswerte :

\begin{tabular}{|c|c|c|c|c|c|c|c|}
\hline BD & $\mathrm{P}$ & $\mathrm{H}$ & $\mathrm{H}-\mathrm{P}$ & $\mathrm{Hg}$ & $P$ & $\mathrm{H}$ & $\mathrm{H}-\mathrm{P}$ \\
\hline $43^{\circ} 728$ & $8 \div 42$ & $8^{\mathrm{m}} \mathrm{I} 8$ & $-0^{m} \cdot 24$ & 42 & $10^{\mathrm{m}} \cdot 97$ & $10^{\mathrm{m}} \cdot 94$ & $-0^{\mathrm{m}} \cdot \mathrm{o}_{3}$ \\
\hline $447 \times 7$ & 8.74 & 8.59 & -0.15 & 49 & I I. 78 & 12.26 & +0.48 \\
\hline 43729 & $8.8 \mathrm{I}$ & 8.52 & -0.29 & 54 & I I.92 & 12.60 & +0.68 \\
\hline 43739 & 9.22 & 9.13 & -0.09 & $5^{6}$ & I 2.32 & I 2.92 & +0.60 \\
\hline 43740 & 9.34 & 9.00 & -0.34 & 65 & I $2.40:$ & I 2.77 & +0.37 \\
\hline $4374 \mathrm{I}$ & 9.44 & $9.5^{I}$ & +0.07 & & & & \\
\hline 43743 & 9.64 & 9.62 & -0.02 & & & & \\
\hline 43735 & 10.33 & $10.3^{1}$ & -0.02 & & & & \\
\hline
\end{tabular}

Die ersten Sterne zeigen noch den normalen negativen Unterschied $\mathrm{H}-\mathrm{P}$, dann folgt zwischen $9^{\mathrm{m}} \cdot 5$ und $\mathrm{I}^{\mathrm{m}}$ eine nahe vollständige Übereinstimmung und zuletzt ein Sprung, dessen Untersuchung mir um so wichtiger schien, als ich meine ganzen visuellen Schätzungen der letzten ${ }_{5}$ Jahre auf der Potsdamer Skala dieser Gegend aufgebaut hatte.

Die nachstehende Sternfolge wurde größtenteils im Jahre IgI9 bẹobachtet. Die Messungen erfolgten an dem bisher benutzten Keilphotometer mit Vergleichstern unter Verwendung des $60 \mathrm{~cm}$-Refraktors, mit dem man bei guter
Luft Sterne $4^{m} \cdot 5$ noch recht bequem messen kann. Als Anschluß dienten einige hellere, sowohl in Potsdam wie in Cambridge gemessene, nach Reduktion auf die Harvardskala gut übereinstimmende Objekte. Zuletzt, nach Fertigstellung der Rechnung, ist zur Vermeidung jeden Zweifels noch eine kleine Kontrollreihe der schwächeren Sterne mit dem Zöllnerschen Photometer durchbeobachtet worden. Das Ergebnis bilden die Tabellen I, II und $V$, von denen die erste auch Farbenangaben enthält. Letztere habe ich, wie bisher, der Osthoffschen Definition anzupassen versucht. Wie zu erwarten stand, hat sich jedoch im Laufe der Zeit eine eigene Skala entwickelt, derart, daß meine Stufe $5^{\mathrm{c}}$ wahrscheinlich bereits dem Grad $7^{\mathrm{c}}$ bei Osthoff entspricht.

$$
\text { I. BD-Sterne }\left(6^{\mathrm{m}}, 5 \text { bis rom } 8\right) \text {, Harvardskala. }
$$

\begin{tabular}{|c|c|c|c|c|c|c|}
\hline Nr. & $\mathrm{BD}$ & $\alpha 1855.0$ & 81855.0 & Beobachtungen & Größe & Fartbe \\
\hline & & $3^{\mathrm{h}}$ & + & & & \\
\hline I & $43^{\circ} 720$ & $17^{\mathrm{m}} 7^{\mathrm{s}}$ & $43^{\circ} \quad 8.6$ & $7.20,37,26,32$ & $7^{\mathrm{m}} \cdot 29$ & $2: 8$ \\
\hline 2 & 43723 & I $73 \mathrm{I}$ & 43. I 2.7 & 8.7 & 8.77 & 2.9 \\
\hline 3 & 43728 & $18 \times 1$ & $43 \quad 5^{2.7}$ & 8.2 & 8.14 & 1.0 \\
\hline 4 & 43729 & 1813 & $43 \quad 49.9$ & 8.6 & 8.59 & 0.6 \\
\hline 5 & 43730 & $18 \quad 27$ & 4352.1 & 6.5 & 6.56 & 4.2 \\
\hline 6 & 43732 & 1845 & $43 \quad$ I 4.7 & 7.19 & $7 \cdot I_{3}$ & 0.9 \\
\hline 7 & 44712 & 1849 & 8.4 & 8.39, & 8.60 & $4 . I$ \\
\hline 8 & 43735 & 2016 & $43 \quad 27.5$ & 9 Messungen & I $0.2 \mathrm{Y}$ & $3 \cdot 4$ \\
\hline 9 & 43737 & 2036 & $43 \quad 14.5$ & $1 \mathrm{x} .00,66,71,72$ & 10.77 & 2.9 \\
\hline
\end{tabular}




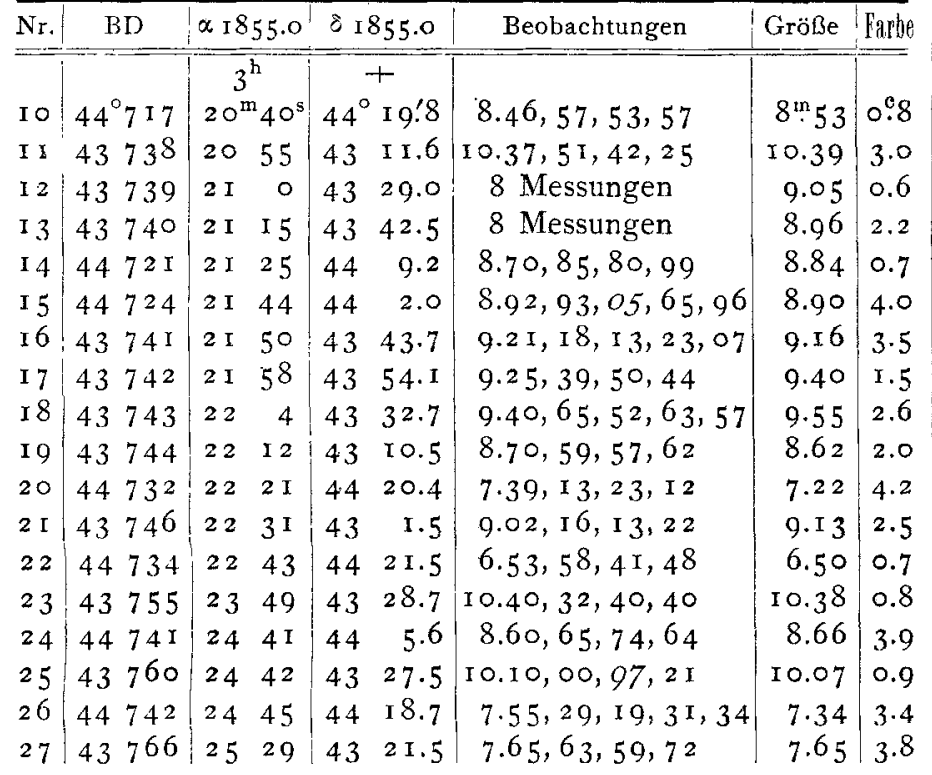

II. Schwache Sterne der Hagenschen Folge ( $\mathrm{I}^{\mathrm{m}}$ bis $\left.\mathrm{I}^{\mathrm{m}}\right)$, Harvardskala.

\section{\begin{tabular}{l}
\hline $\mathrm{Nr}$ \\
\hline 28 \\
29 \\
3 \\
3 \\
3 \\
33 \\
34 \\
3 \\
3 \\
3 \\
3 \\
3 \\
4 \\
4 \\
4 \\
4 \\
4 \\
4
\end{tabular}}

\begin{tabular}{l|l|l|l|l|}
\hline $\mathrm{Nr}$. & $\mathrm{Hg}$ & $\alpha$ I 855.0 & $\delta$ I 855.0 & Beobachtungen \\
\hline
\end{tabular}

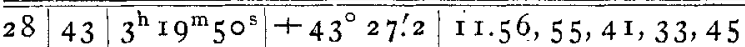

$2946 \quad 32000+43 \quad 30.5$ I 2.33 , I $5,26,30$

30 5 I 3 20 5 5 43 27.2 9 Messungen

\begin{tabular}{l|l|lll|ll|l}
31 & 64 & 3 & 20 & 37 & +432 I.5 & I $3.00,9 I, 98,98$
\end{tabular}

$3^{2} 5^{8} 32037+4323.4$ × $2.65,78,86,87,83$

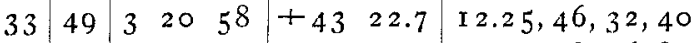

$3454 \quad 32$ I I +4320.3 I.2.7 I, 78, 76, 82

$355632 \mathrm{r} 4+4326.2 \quad 8$ Messungen

$3678 \quad 32$ I $8+4325.8$ I $3.77,77,82,69$

$3780 \mid 32$ I 16 + 43222.2 т $4.13,04,06,18,97$

$3^{8} 44,32$ I $22+43$ I 3.3 I I.29, $35,38,54$

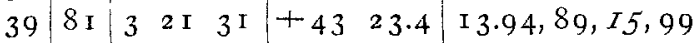

40,7732 I $35+4325.3$ I $3.72,80,7$ I, 92

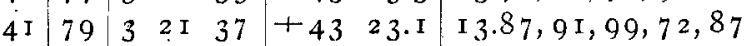

$4274 \quad 3$ 2I $43+4325.8$ I $3.51,62,72,43$

435032 I $53+4335.6$ I $2.59,8$ I, 62,74

$446632 \mathrm{I} 55+4320 . \mathrm{I}$ I $3.01,95,74,71$

$45: 68\left|\begin{array}{lll}3 & 21 & 56\end{array}\right|+4325.7 \mid$ I $2.82,67,04,74,67$

\begin{tabular}{|c|c|c|c|c|c|}
\hline $\mathrm{Nr}$ & $\mathrm{Hg}$ & $\alpha_{\mathrm{I}} 855.0$ & 81855.0 & Beobachtungen & Gr. \\
\hline 46 & 42 & $3^{\mathrm{h}} 2 \mathrm{I}^{\mathrm{m}} 57^{\mathrm{s}}$ & $+43^{\circ} 24 \cdot 4$ & $10.88,91,05,94,96,07$ & $10^{\mathrm{m}} \cdot 97$ \\
\hline 47 & 65 & $\begin{array}{lll}3 & 22 & 4\end{array}$ & +4329.0 & I $3.05,96,85,88$ & I 2.94 \\
\hline 8 & 47 & $322 \quad 16$ & $+433^{6 . I}$ & I $2.25,47,42,3 \mathrm{I}$ & I 2.36 \\
\hline 40 & 48 & 22 & $+43 \times 9.2$ & I $2.33,25,18,33$ & 12.27 \\
\hline
\end{tabular}

III. Vergleichung der Bergedorfer Werte mit der Potsdamer Skala. $(B=$ Bgdf., $P=$ Potsd.)

\begin{tabular}{|c|c|c|c|c|c|c|c|}
\hline $\begin{array}{l}\text { Nr. } \\
22\end{array}$ & $\begin{array}{c}\mathrm{B} \\
6^{\mathrm{m}} \cdot 5^{\circ}\end{array}$ & $\begin{array}{c}\mathrm{P} \\
6 ! 6 \mathrm{I}\end{array}$ & $\begin{array}{c}\mathrm{B}-\mathrm{P} \\
-\mathrm{O}^{\mathrm{m}} \mathrm{I} \mathrm{I}\end{array}$ & $\begin{array}{l}\mathrm{Nr} . \\
\mathrm{I} 7\end{array}$ & $\begin{array}{c}\mathrm{B} \\
9^{\mathrm{m}} \cdot 40\end{array}$ & $\begin{array}{c}P \\
9^{m} \cdot 34\end{array}$ & $\begin{array}{c}\mathrm{B}-\mathrm{P} \\
+0.06\end{array}$ \\
\hline 5 & 6.56 & 6.57 & -0.01 & 18 & 9.55 & 9.64 & -0.09 \\
\hline 6 & 7.13 & 7.44 & $-0.3 \mathrm{I}$ & 25 & 10.07 & 10.00 & +0.07 \\
\hline 0 & 7.22 & 7.16 & +0.06 & 8 & I 0.21 & 10.33 & -0.12 \\
\hline I & 7.29 & $7 \cdot 3^{6}$ & -0.07 & 23 & $10.3^{8}$ & 10.26 & +0.12 \\
\hline 26 & $7 \cdot 34$ & $7.5^{\circ}$ & -0.16 & 46 & 10.97 & 10.97 & 0.00 \\
\hline 7 & 7.65 & 7.89 & -0.24 & 28 & I 1.46 & I 1.08 & +0.38 \\
\hline 3 & 8. I 4 & 8.42 & -0.28 & 48 & 12.36 & I I. 83 & +0.53 \\
\hline 10 & 8.53 & 8.74 & -0.21 & 33 & I 2.36 & I 1.78 & +0.58 \\
\hline 4 & 8.59 & $8.8 \mathrm{I}$ & -0.22 & 30 & I 2.64 & 12.00 & +0.64 \\
\hline 7 & 8.60 & 8.92 & -0.32 & 35 & I 2.68 & 12.32 & +0.36 \\
\hline 9 & 8.62 & 8.80 & -0.18 & 43 & I 2.69 & 12.20 & +0.49 \\
\hline 4 & 8.66 & 9. I 0 & -0.44 & 34 & I 2.77 & 11.92 & +0.85 \\
\hline 2 & 8.77 & 9.07 & -0.30 & 45 & I 2.79 & I $2.3 \mathrm{I}$ & +0.48 \\
\hline I 4 & 8.84 & 9.03 & -0.19 & 47 & I 2.94 & I $2.40:$ & +0.54 \\
\hline 5 & 8.90 & 9.16 & -0.26 & 42 & I 3.57 & I $2.62:$ & +0.95 \\
\hline I 3 & 8.96 & 9.34 & $-0.3^{8}$ & 40 & I 3.79 & I 2.77: & +1.02 \\
\hline I 2 & 9.05 & 9.22 & -0.17 & 41 & I 3.87 & r $2.85:$ & +1.02 \\
\hline ? & 9.13 & 9. I 4 & -0.01 & 39 & I 3.99 & 13.08 & $+0.9 \mathrm{I}$ \\
\hline 6 & 9.16 & 9.44 & -0.28 & 37 & 14.08 & $\mathrm{I} 3 \cdot 3 \mathrm{I}:$ & +0.77 \\
\hline
\end{tabular}

IV. Vergleichung der Bergedorfer Werte mit der Harvardskala. $(\mathrm{B}=$ Bgdf., $\mathrm{H}=$ Harv. $)$

\begin{tabular}{|c|c|c|c|c|c|c|c|c|}
\hline 12.36 & \multicolumn{8}{|c|}{ Harvardskala. $(\mathrm{B}=$ Bgdf., $\mathrm{H}=$ Harv. $)$} \\
\hline 12.77 & Nr. & $\mathrm{B}$ & $\mathrm{H}$ & $\mathrm{B}-\mathrm{H}$ & $\mathrm{Nr}$. & B & $\mathrm{H}$ & $\mathrm{B}-\mathrm{H}$ \\
\hline I 2.68 & 22 & $6^{\mathrm{m}} \cdot 50$. & $6^{m} \cdot 45$ & $+0^{\mathrm{m}} .05$ & I 2 & $9^{m} \cdot \circ 5$ & $9^{\mathrm{m}} \cdot \mathrm{I}_{3}$ & $-0 \mathrm{mos}$ \\
\hline 13.76 & 5 & 6.56 & $6.9 \mathrm{I}$ & -0.35 & I 6 & 9.16 & $9 \cdot 5^{I}$ & -0.35 \\
\hline I 4.08 & 6 & 7.13 & 7.25 & -0.12 & I 8 & 9.55 & 9.62 & -0.07 \\
\hline I I. 39 & 20 & 7.22 & $7 \cdot 3^{2}$ & -0.10 & 8 & 10.21 & $10.3 \mathrm{I}$ & -0.10 \\
\hline 13.99 & I & 7.29 & 7.28 & +0.01 & 46 & 10.97 & 10.94 & +0.03 \\
\hline I 3.79 & 26 & $7 \cdot 34$ & $7 \cdot 76$ & -0.42 & 29 & 12.26 & I 1.86 & +0.40 \\
\hline I 3.87 & 27 & 7.65 & $7 \cdot 7 \mathrm{I}$ & -0.06 & 33 & I 2.36 & I 2.26 & +o.10 \\
\hline 3.57 & 3 & 8.14 & 8. 18 & -0.04 & 47 & I 2.94 & I 2.77 & +0.17 \\
\hline I 2.69 & IO & 8.53 & 8.59 & -0.06 & 35 & 12.68 & $\times 2.92$ & -0.24 \\
\hline I 2.85 & 4 & 8.59 & $8.5^{2}$ & +0.07 & 44 & I 2.85 & I 2.93 & -0.08 \\
\hline
\end{tabular}

V. Kontrollmessungen am Zöllnerphotometer. (I920 Febr. 23 und März 24.)

\begin{tabular}{|c|c|c|c|c|c|c|c|c|c|c|c|}
\hline $\begin{array}{r}\mathrm{Nr} . \\
8\end{array}$ & $\begin{array}{l}\text { Beobachtungen } \\
9.87, I_{4}, O O\end{array}$ & $\begin{array}{l}\text { Gr. } \\
\text { I } 0^{\mathrm{m}} .00\end{array}$ & $\begin{array}{l}\text { Diff. } \\
+0.2 I\end{array}$ & $\begin{array}{l}\text { Nr. } \\
35\end{array}$ & $\begin{array}{l}\text { Beobachtungen } \\
12.67,65,65\end{array}$ & $\begin{array}{l}\text { Gr. } \\
12^{\mathrm{m}} \cdot 66\end{array}$ & $\begin{array}{c}\text { Diff. } \\
+0^{\mathrm{m}} .02\end{array}$ & $\begin{array}{l}\mathrm{Nr} . \\
40\end{array}$ & $\begin{array}{l}\text { Beobachtungen } \\
\text { I } 3.5 \mathrm{I}, 60\end{array}$ & $\begin{array}{l}\text { Gr. } \\
\text { I } 3^{m} \cdot 56\end{array}$ & $\begin{array}{l}\text { Diff. } \\
+0.23\end{array}$ \\
\hline 46 & Anschlußstern & $x 0.96$ & +0.01 & 34 & I $2.75,80$ & I 2.78 & $-0.0 \mathrm{I}$ & $4 \mathrm{I}$ & I $3.53,75,65$ & I 3.64 & +0.23 \\
\hline 28 & I I.I 6,39 & I 1.28 & +0.18 & 45 & $\mathrm{I} 2.94, O I$ & 12.98 & -0.19 & 39 & I $3.70,84$ & 3.77 & +0.22 \\
\hline 33 & I $2.35,52$ & I 2.44 & -0.08 & 44 & I $2.94, I 0$ & I 3.05 & -0.20 & 37 & I $4.05, \circ 7,02$, I 3 & 14.07 & +0.01 \\
\hline 30 & I $2.55,58$ & $12.5^{6}$ & +0.08 & $4^{2}$ & I $3.46,42$ & 13.44 & +0.13 & & & & \\
\hline
\end{tabular}

Wie aus den Differenzen der Ergebnisse in I und II gegen V hervorgeht, besteht wohl ein geringfügiger systematischer Unterschied zwischen den Messungen mit dem Keil und mit dem Polarisationsphotometer. Der Widerspruch zwischen der Potsdamer und der Harvardreihe wird aber attch hier im Sinne der letzteren entschieden.

K. Graff.

Notiz. Das vor dem Kriege errichtete Privatobservatorium in Nixdorf, Böhmen, ersucht die einzelnen Sternwarten um freundliche Überlassung ihrer Veröffentlichungen, da dieselben im Buchhandel nur zu für ein Privatunternehmen unerschwinglichen Preisen erhältlich sind. Mag. Adolf Krause. 